\title{
Multi Object and Dynamic Query Based CBIR System using DCT Incorporated with HOG and HTF
}

\author{
G. Suresh, N.C. Sendhil Kumar, R.Murugesan, P.Mukunthan
}

\begin{abstract}
This work contributes multi object detection and dynamic query image based retrieval system. Generally, finding relevance and matching user expectations is very critical based on query key information and these results irrelevant responses which will produce low similarity index. Consequently, CBIR system took a major responsibility of identifying new objects, retrieving similar objects or contents based on multi query and dynamic keywords with improved recall and precision as per requirement of the users. At this juncture, Discrete Curvelet Transform with the incorporation of HOG and HTF based approach is proposed to handle commercial image, medical images and types of multi model images. This proposed approach mainly focuses on extracting scaled features for finding correlation among the query and database images. To start with the process, query image is decomposed into multi level sub images to extract set of texture features at two levels. These features are estimated by Gray Level Co-occurrence Matrix (GLCM) and HOG descriptor based techniques is adapted to find scaled vectors with reduced dimensionality. This method outperform compared as compared to existing method is authenticated from experimental results.
\end{abstract}

Keywords: image retrieval, HOG, curvelet transforms, GLCM

\section{INTRODUCTION}

With the advent of the digital technology, there is various form information with larger scale accessed in different types search engine. Timely data collection and more relevant data collection is one of the primary processes of retrieval system. Content Based Image Retrieval (CBIR) is the key component for availing instant resourceful data in the search engine, which is another thirst area in research scope [1], [2]. The basic step involved in CBIR is retrieving highly correlated images from database by feeding query image based on relevance. Practically, CBIR involves the contents like, texture, shape or color based searches. Therefore, feature selection is a key stage in content-based retrieval [3], [4]. Figure 1 describes the general process and operation of CMIR system. There are plenty of sources of information are accumulated in database and its profiles are maintained as feature vector during preprocess [5][6][8]. When query image is given as an input, its feature values are estimated and are compared with the database feature with some statistical measures. Then, based on rank from higher

Revised Manuscript Received on 14 August, 2019.

G. Suresh, N.C., Professor, Sri Indu College of Engineering and Technology, Hyderabad, Telangana, India.

Sendhil Kumar, Professor, Sri Indu College of Engineering and

R.Murugesan, Associate Professor, Annamacharya Institute of Technology \& Sciences, Tirupathi, AndhraPradesh, India.

P.Mukunthan, Professor, Sri Indu College of Engineering and Technology, Hyderabad, Telangana, India. Technology, Hyderabad, Telangana, India.

similarity index to the lower similarity index retrieved images are ordered.

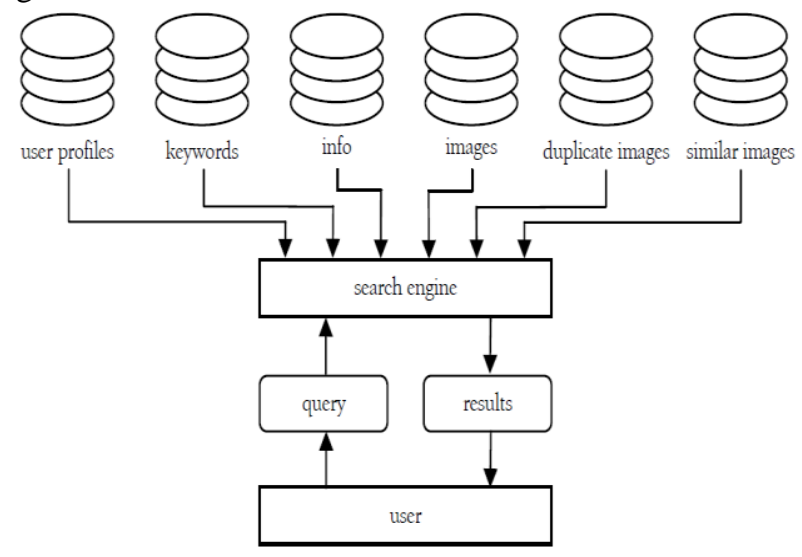

Figure 1 General CBIR system

\section{RELATED WORK}

Juan Miguel Medina et al (2012) proposed a novel approach to medical image retrieval using a fuzzy objectrelational database management system (FORDBMS). This paper concentrates on X-ray images of patients suffering from scoliosis (a medical condition in which the patient's spine is curved) from which spine descriptions are obtained. Subsequently, query images are matched to retrieve relevant images with a certain oriented pattern. Results show high accuracy when evaluated by medical experts. A comparison study with other dynamic CBIR systems, the revealed work here is domain independent, flexible, and highly scalable.

SubrahmanyamMurala et al (2012) mitigated LTrP method for CBIR system. The proposed approach encompasses the connectivity between the current pixels to its surrounding pixels, based on the orientation of the image objects the movements of the feature vectors are evaluated using the differentiation scheme in $\mathrm{X}-\mathrm{Y}$ directions. The efficiency of the proposed scheme is compared with LBP, Gabor filter and LTP based approaches and the synthesized results tested with benchmark data.

\section{METHODOLOGY}

DCT with the incorporation of HOG and HTF based approach is proposed to handle commercial image, medical images and types of multi model images. This proposed approach mainly focuses on extracting scaled features for finding correlation among the query and database images. 
To start with the process, query image is decomposed into multi level sub images to extract set of texture features at two levels. These features are estimated by Gray Level Cooccurrence Matrix (GLCM) and HOG descriptor based techniques is adapted to find scaled vectors with reduced dimensionality. The proposed method is evaluated with performance measures and also compared with conventional techniques.

Figure 2 depicts the architecture of proposed Method as shown below.

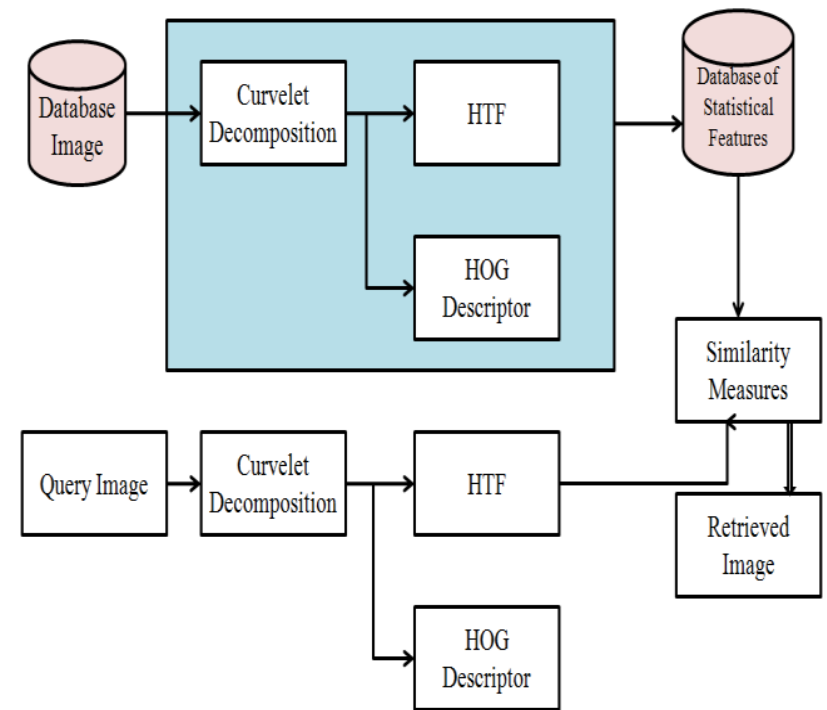

Figure 2 Architecture of Proposed DCT incorporated with HOG and HTF

Proposed Algorithm for Retrieval Process

Step 1: Image is decomposed using curvelet.

Step 2: The HOG descriptor is introduced for extracting feature vector.

Step 3: Also, Statistical HTF Features are calculated.

Step 4: Obtain five features for query image and HOG descriptions

Step 5: Compare the query image and database images from the calculated statistical feature vectors for similarity measure.

Step 6: Rearrange the features to evaluate correlation index.

Step 7: Top Closest images are ranked in GUI

\section{SIMULATION RESULTS}

This CBIR system is developed in MATLAB 7.5 environment. The extensive experimental study has been made to demonstrate CBIR system for various form query images. In our proposed study color, texture or shape based evaluation made. There three types of images were taken (commercial images, medical images, group images) for identifying the strength of the retrieval system [8-13]. Totally 1500 images, from each type 500 images were maintained in the database, which are all environment independent collections. The minimum number of statistical feature vectors is 7500 for database images. Then, based on query input features correlation index is calculated and every score is maintained for the identification of future relevance. Figure 3 details the commercial query image and its relevance with all statistical features. Similarly, medical image is considered to test the performance with all conditions as shown in figure 4. GUI is developed to present the overall process of entire scheme.

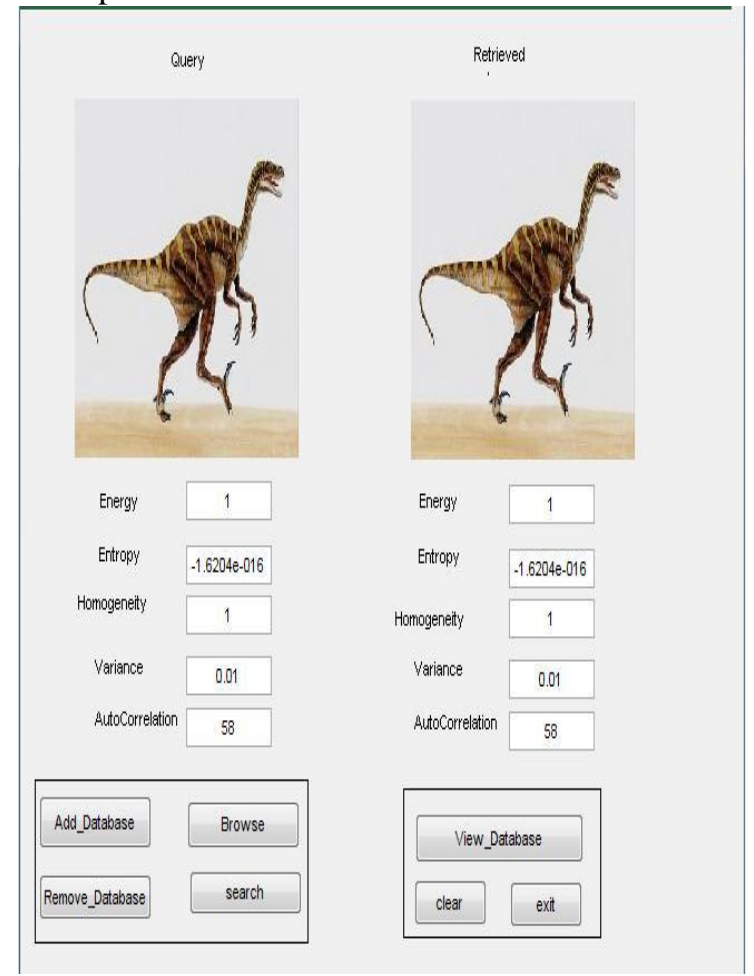

Figure 3 Retrieval Process for Commercial Images

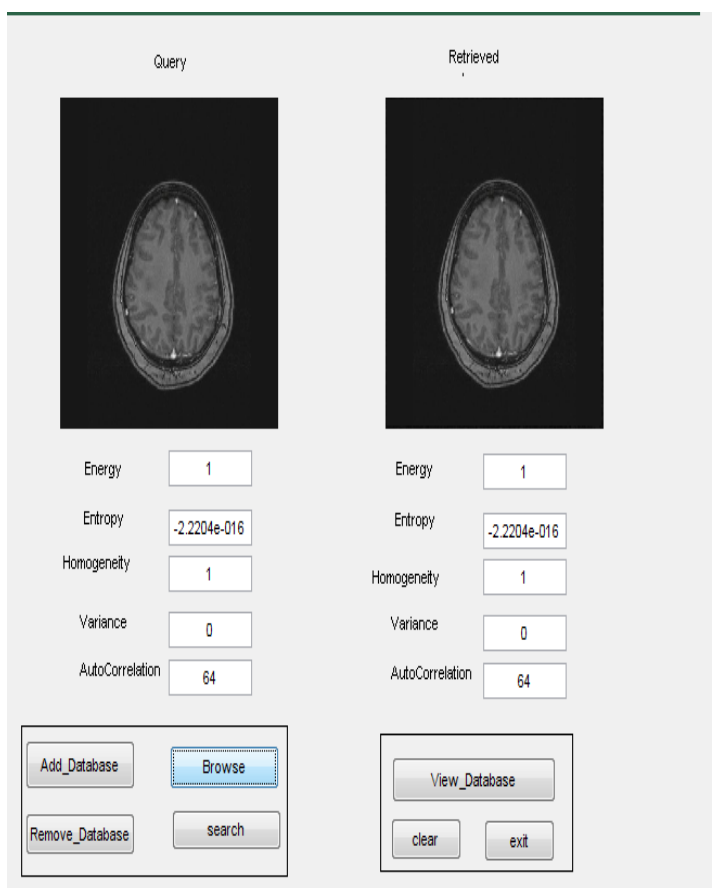

Figure 4 Retrieval Process for Medical Images

From the collection of images classification is done with the closest image to the approximated image and also uncorrelated images. By analyzing the recall rate and precision, the efficiency of classification is defined. The summary of recall and precision values are plotted in figure 5. Another benchmark image is taken for experimenting

Published By: Blue Eyes Intelligence Engineering \& Sciences Publication 
multi query image retrieval and is demonstrated. Hence, the proposed system performs better than other Gabor and Wavelet based methodologies. Figure and Figure 7 reveals the multi object detection for the multi object query images.

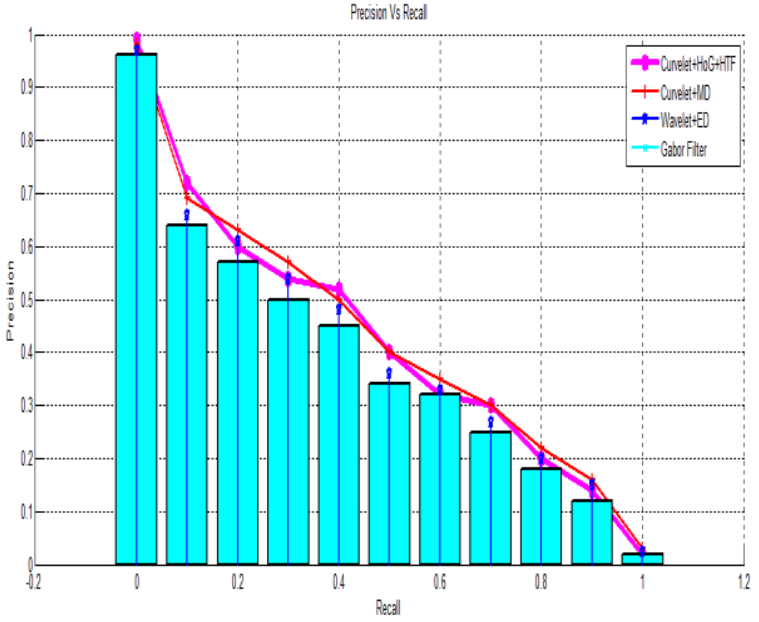

Figure 5 Plots between Recall and Precision
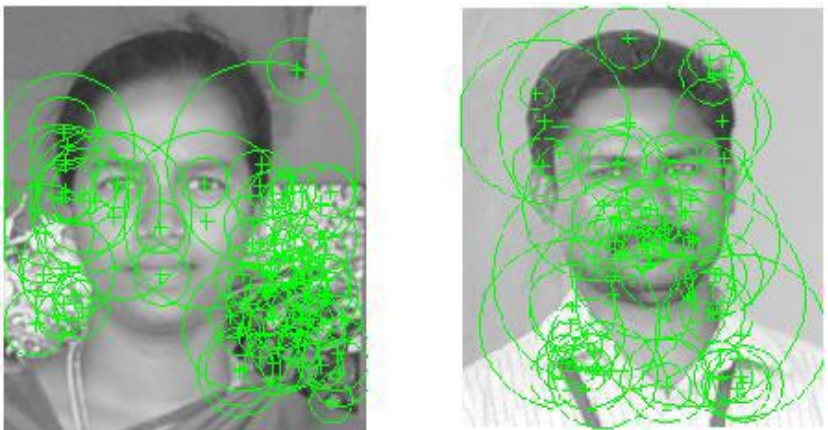

Figure 6 Multi query and multi model query Images

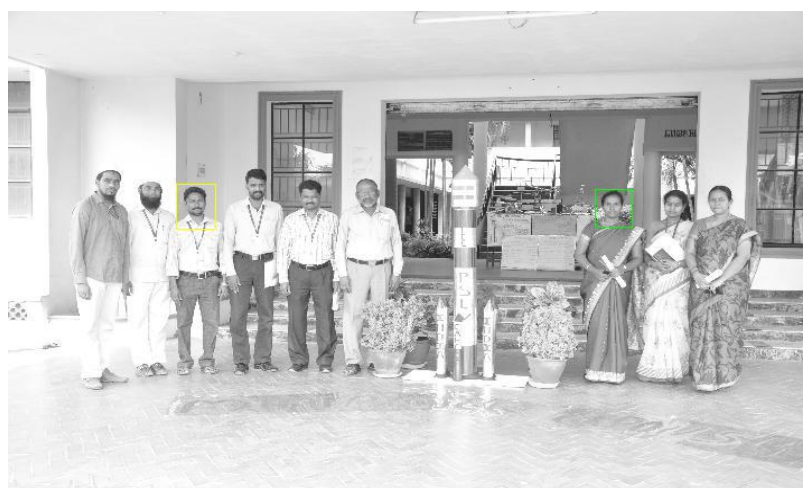

Figure 6 Multi object detection and retrieval process from query Images

\section{APPLICATIONS}

CBIR system is effectively implemented in district science exhibition for students' level demonstration. There are two types of analysis were made; one is by showing wooden physical pattern as a query template then retrieves related similar patterns and history of those properties; secondly by touching the property the related similar objects and correlated contents were retrieved. Also this is been implemented to track and detect feature based multiple objects for multi query inputs.

\section{CONCLUSION AND FUTURE ENHANCEMENT}

Hence, Discrete Curvelet Transform with the incorporation of HOG and HTF based approach is proposed to handle commercial image, medical images and types of multi model images. This proposed approach mainly focused on extracting scaled features for finding correlation among the query and database images. These features are estimated by Gray Level Co-occurrence Matrix (GLCM) and HOG descriptor based techniques is adapted to find scaled vectors with reduced dimensionality. This method outperform as compared to existing method is authenticated from experimental results. Further, this work can be strengthened by mitigating huge and dynamic query data, which will offer most sensitive features for the critical combinations of key inputs. Also, this approach can be enhanced for duplication detection classification and to improve robustness.

\section{REFERENCES}

1. Yangxi Li, et al., (2012), "Difficulty Guided Image Retrieval Using Linear Multiple Feature Embedding IEEE Transactions on Multimedia, Vol. 14, No.6.

2. Xiaoou Tang et al., (2012), "Intent Search: Capturing User Intention for One-Click Internet Image Search", IEEE Transactions on Pattern Analysis And Machine Intelligence, Vol. 34, No. 7.

3. Tatsuki Murakami et al., (2012), "Features for image retrieval: the impression degree of a human image by overexposure occurring in the facial area", SICE Annual Conference,August 20-23, Japan.

4. Ben-Haim et al., (2006), "Improving Web- Based Image Search via Content Based Clustering," Proc. Int'l Workshop Semantic Learning Applications in Multimedia.

5. Yuan-feng et al., (2012), "Image Retrieval Using ESNs and Relevance Feedback", 11th International Symposium on Distributed Computing and Applications to Business, Engineering \& Science

6. Josip et al., (2010), "Improving Web Image Search results using query relative classifiers" IEEE 9784244-6985-7/10.

7. Chen Caol et al., (2012), "Online - Non feedback image Re-ranking via dominant data selection", MM'12, October 29-November 2, Japan.Copyright 2012 ACM 978-1-4503-1089-5/12/10.

8. Arulananth et al., (2017), "Fake shadow detection using local histogram of oriented gradients (HOG) features", 2017 International conference of Electronics, Communication and Aerospace Technology (ICECA), Vol.2, pp.739 - 742 .

9. J. Cui, F. Wen, and X. Tang, (2008), "IntentSearch: Interactive OnLine Image Search Re Ranking," Proc. 16th ACM IntlConf.Multimedia, 2008.

10. Zhao et al., (2015), "An Enhanced HoGFor Pedestrian Detection", IEEE Intell. Transp. Syst. Mag. Vol. 7,pp. 29-38.

11. YongHong Zhang. (2011) "Digital image hiding using curvelet transform", IEEE International Conference on Computer Science and Automation Engineering.

12. Suoqi Zhang et al.,(2017) "Sparsity-motivated multi scale histograms of oriented gradients feature for SRC", IEEE International Conference on Unmanned Systems (ICUS),pp.389-393.

13. Arulananth et al., (2017), "Fake shadow detection using local histogram of oriented gradients (HOG) features", 2017 International conference of Electronics, Communication and Aerospace Technology (ICECA), Vol.2, pp.739 - 742 